\title{
Design of Data Communication Network and Communication Software in Rural Substations
}

\author{
Q.C. CHEN, H. LI, B. LI, C. LIU, J.Y. LIN, L. CAI \& L. XIA \\ China Electric Power Research Institute, Beijing, China
}

\begin{abstract}
Combining with integrated Substation Automation Systems, this paper describes the data communications and the development trends of the Substation Automation Systems, which is suited to the new or modified rural substations. The paper also presents the design methods of the Supervisory Control and Data Acquisition (SCADA) software, which can meet a variety of interface types and protocols.
\end{abstract}

KEYWORD: Rural electric network; Substation automation; Data communication network

\section{GENERAL INSTRUCTIONS}

The voltage level of rural substations are mainly $110 \mathrm{kV}$ or less. For new construction and renovation of substation automation systems, there are two main design modes [1-2]: distributed RTU (Control Unit) with conventional protected and hierarchical distributed system. To reform substation with complete secondary equipment and protective equipment, conventional RTU with protected mode is often to be used. To build substation or to reform the substation with secondary equipment running poorly, hierarchical distributed integrated automation system is often to be used. In this paper, taking integrated substation automation system as an example, focuses on hierarchical distributed integrated automation system station data communications, as well as communication software design methods.

\section{COMMUNICATION NETWORKS IN A SUBSTATION}

The substation data communication network is the key of substation integrated automation system. It is a bridge to exchange information within the station between the various subsystems or functional modules, and it is also an essential part for substation monitoring and control.

\subsection{Communication technology in a substation}

The communication technology are used mainly as the following: serial communication technology, fieldbus technology and Ethernet communication technology [3].

As its low transmission efficiency, transmission distance and the expansion difficulties, serial communication technology can only be as a secondary means of communication in the substation automation system basically.

Based on Network Technology, fieldbus communication technology with flexible networking has obvious advantages over the serial communication technology in terms of communication rate, real-time and reliability, and soon became the major communication technology in the substation automation system. CAN bus [4] and LonWorks [5] are the two widely used manners of fieldbus communication technology, but there are still some deficiencies:

(1) When communication nodes exceeds a certain number, the response rate drops quickly;

(2) When the transmission data is large, there is the transmission delay;

(3) As the interface of communication nodes does not have uniform standards, general equipment manufacturers only support their own communication nodes.

The Ethernet communication technologies have obvious advantages in terms of bandwidth, communication media, topology, etc., in particular, Ethernet communication by fiber optic with antielectromagnetic interference capability and high transfer rate, making Ethernet communication technology become the development trend of substation automation technology. 


\subsection{Communication network in a substation}

According to the functions Substation automation system can be divided into the station control level, spacer level and process level logically. The process level is the connection system between the primary equipment and bay Equipment, such as input/output, smart sensors and controllers, etc. As its communication does not have the traditional sense, communication here does not include the process level. Integrated Substation Automation System is a hierarchical distributed system, divided into bay level and station control level. Bay level devices consists of real-time information monitoring unit, control unit, protection unit, etc., to realize real-time monitoring, information collection, an equipment protection, control and other functions. Station level device consists of station level computers and communication master control unit, which is the center of the station's equipment monitoring, measurement, control, management, and centralized control center, simultaneously with the centralized control center and the dispatching end for remote data communication.

According to the communication technology used between bay level and station control level and division for each station level device, two models of typical station communication networking are designed:

1) Three layer information exchange mode based on CAN bus

As shown in Figure 1, using three layer network equipment: information exchange by bay layer equipment transferred to the communication management machine, and then the communication management unit transferred it to the local monitoring system.

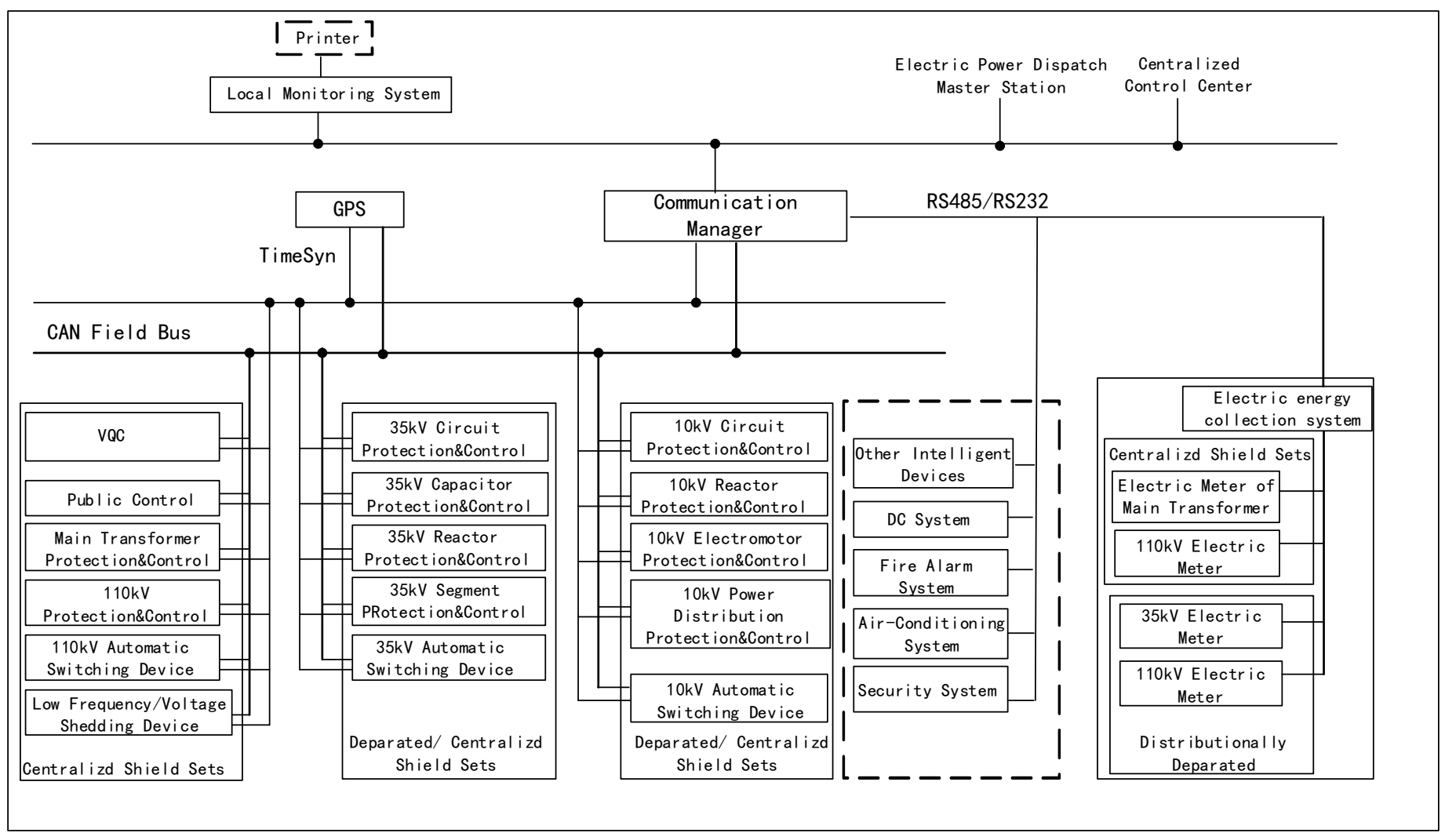

Figure 1. Three layer information exchange based on CAN bus

Communications between bay level equipment and management units mainly dominated CAN bus. Other equipment that cannot communicate with communications manager by the CAN bus, such as DC systems, security, power meter screen, as well as other intelligent devices, can communicate by RS485 or RS232.Communication manager communicate with the local monitoring system can use Ethernet or RS232.

\section{2) Tow layer information exchange mode based on Ethernet}

As shown in Figure 2, using three layer network equipment: information exchange by bay layer protect and control unit transferred to the communication management machine, and simultaneously transferred to the local monitoring system. Most equipment of bay level access to the LAN directly, and for the equipment cannot access to the LAN, can be communicated with the communication manager first, then access to the local area network by the communication manager. 


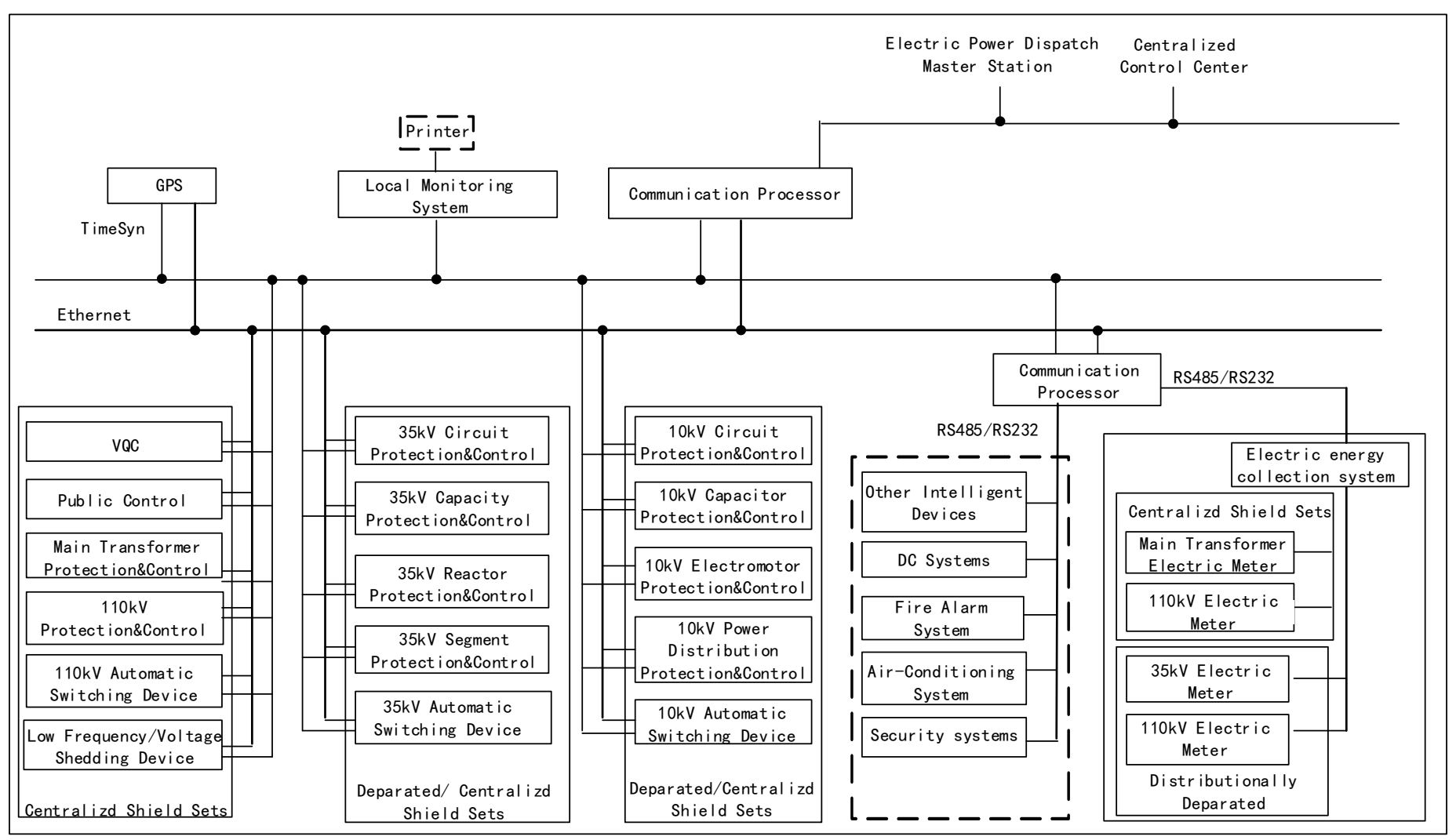

Figure 2. Tow layer information exchange mode based on Ethernet

\section{DESIGN AND IMPLEMENTATION OF COMMUMICATION SOFTWARE}

Communication of the rural substation automation systems has its own peculiarities: a variety of networking mode and processing multiple protocols simultaneously, which requires communication software design should be flexible configuration and easy expansion.

The main task of the substation communication software is to communicate with the terminal device, preprocess the received packets, store the interpretation of data in the database, or out on to the background service, transfer the data to the terminal communication node initiated by the background HMI, such as setting data, remote control, etc.

\subsection{The design idea of the software}

The software uses the object-oriented design method, modular design, make the whole software flexible configuration, convenient to extend, is easy to use and easy to maintenance.

Data Stream of the communication software layering parsed by two layers: link layer and application layer. The link layer is mainly processing all kinds of communication interface, including communication port initialization, the rawdata reading and writing. The application layer parses the messages from the link layer in accordance with the protocol of agreement, and then progresses the parsed data if necessary, at the same time, organizes downlink packets according to the agreement.

With main standby switching function, communications software can automatically substitute host using hot standby host, continue to communicate with the terminal, when there are multiple background computers, hosts shut down or malfunction.

With interface inspection function, communication software can re-initialize the communication interface, when the communication interface is abnormal, without affecting the normal communication with other communication port.

\subsection{Object model of the software}

Communication software using the universal object modeling method, designed classes as follows: communication protocol base class CProtocol, communication interface base class CCommBase, and subclasses by these two base classes derived. Figure 3 is a graph of the protocol class inheritance, and Figure 4 is a diagram of communication interface inheritance class. 


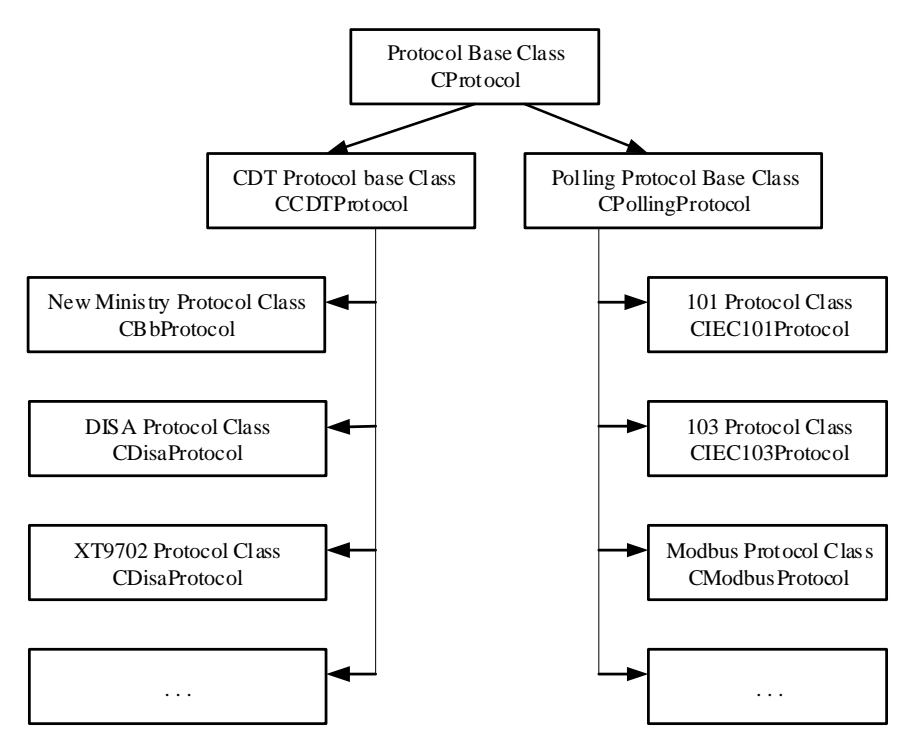

Figure 3. Protocol class inheritance diagram

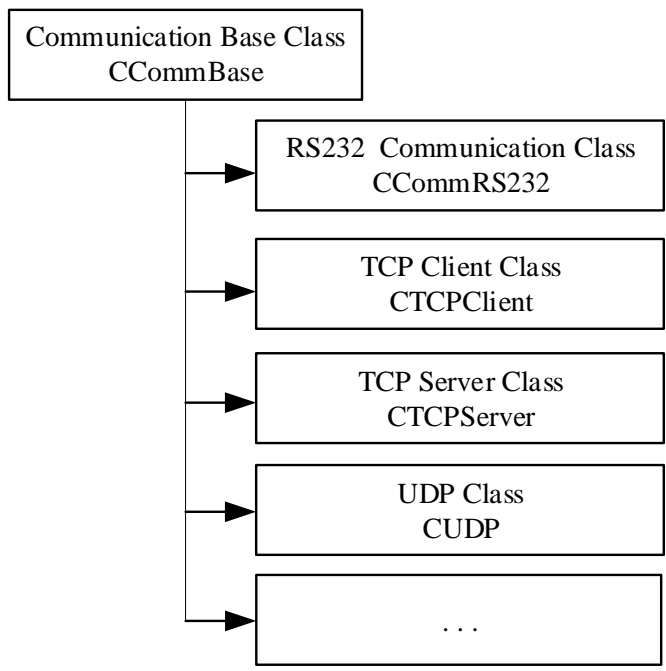

Figure 4. Communication class inheritance diagram

\subsection{Software implementation}

As shown in figure 5, communication software is composed of main and standby switching thread, communication main thread, interface polling thread, Run log and packets saving thread, interface display thread, etc. Main and standby switching thread can monitor the status of the host and the hot standby host, and would promotes the hot standby host to the host after the host exits; Communication main thread finishes reading data from communication interfaces, packet parsing, data processing, command issuing, etc; Interface polling thread is responsible for checking the current status of various communication interfaces and notify the main thread to re-initialize the interface object when the interface is abnormal; Run log and packets saving thread is responsible for recording system operation log and storing packets sent or received, providing information to support comprehensive debugging and troubleshooting; Interface display thread can show the original packets in the process of communication and the specific interpretation of the packets.

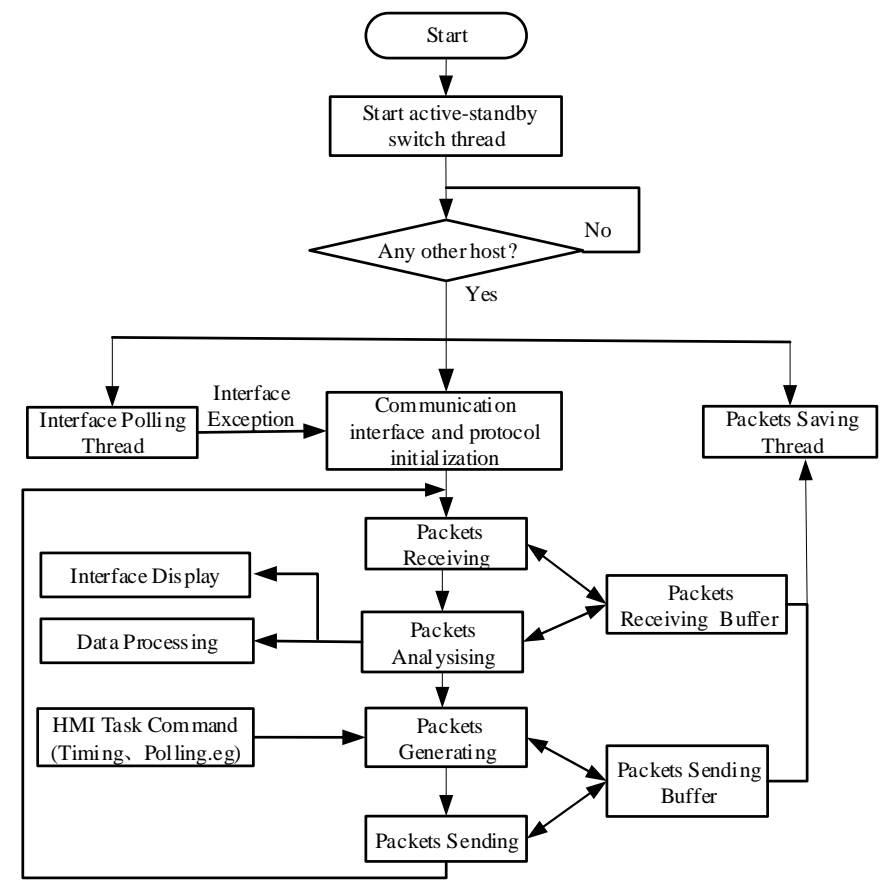

Figure.5 Communication program flow chart

As the core of the communication software, the main thread of communication is an important part of achieving specific communication interfaces and communication agreement. Message parsing of application layer uses the way of data flow stratified analysis. According to the maturity and complexity, the data analyzed by communication protocol packets, can be divided into four types: real-time data, historical data, functional data and transparent transmission of data.

Real-time data completely representing the information of analog quantity or state quantity, can be updated immediately, storage or tips after parsed by the communication protocol.

Relative to the real-time data, historical data is a group of data or files with specific meanings. The data would not make a detailed analysis on the process of communication software, but referred to the other service thread or process for the further analysis. For example, there is no sense to analysis the disturbance data in IEC60870-5-103 separately. Only after collected completely, the noisy data is useful.

Functional data is temp data to guarantee smooth and specification of the communication process. The data are generally used only within the application level.

Transparent transmission data is the communication data to inclusive communication node in the next level according to the protocol. It is also a method to upload the communication protocol packets. At present, the most common way to upload the protection protocol packages (such as LFP, NZ94, etc.) is by extending the CDT or IEC60870-5103. When parsing the packets, the application layer protocol restores these packets according with the 
packaging protocol uniform, and then delivered it to the protocol pool for further parsing.

\section{CONCLUSION}

The communication network in a substation and the background monitoring communication software described by this paper has been widely used in the national rural substations. Proved by a number of field operation, with a clear structure the system is stable and reliable.

Using modeling methods and object-oriented hierarchical data flow analysis technology, it applied the flexible features of inheritance and polymorphism, making it more suitable for the communication in diverse communication network modes in a substation, has brought great convenience for system installation, debugging, as well as operation and maintenance of the back-end. Function expansion later is also very convenient.

\section{REFERENCES}

[1] TIAN Guo-zheng. 2003. Selection of communication network and protocol for substation automation system. Power System Technology 27(9): 66-68.

[2] Xiang, GAO, etc. 2006. Main Features and Key Technologies of Digital Substation. Power System Technology 30(23):67-71, 87.

[3] Ye Lei. 2006. Research on substation automation system communication structure and protocols. North China Electric Power University: Master.

[4] Chen Jingqian. 2005. The design of substation monitoring and control system based on CAN bus. South China University of Technology: Master.

[5] Gao-xiong, HU etc. 2005. Development of Substation Automation Based on the LonWorks Control System Technology. Proceedings of The Electric Power System And Automation. 17(1):83-87. 